\title{
Vibrational Investigation of DODC Cation for Recognition of Guanine Dimeric Hairpin Quadruplex Studied by Satellite Holes
}

\author{
Ji-Yen Cheng, ${ }^{\dagger,+}$ Sheng-Hsien Lin, ${ }^{\dagger,+}$ and Ta-Chau Chang* $*$ \\ Institute of Atomic and Molecular Sciences, Academia Sinica, P.O. Box 23-166, \\ Taipei, 10764, Taiwan, Republic of China, and Department of Chemistry, National Taiwan University, \\ Taipei, 10764, Taiwan, Republic of China
}

Received: December 18, 1997; In Final Form: April 28, 1998

\begin{abstract}
We have introduced the satellite hole spectral method to examine the binding sites of 3,3'-diethyloxadicarbocyanine cation $\left(\mathrm{DODC}^{+}\right)$to various guanine-rich oligonucleotides. The satellite hole patterns along with normal mode calculations allow us to determine the interaction of $\mathrm{DODC}^{+}$with a dimeric hairpin quadruplex formed by sequence $d\left(\mathrm{G}_{4} \mathrm{~T}_{4} \mathrm{G}_{4}\right)$. Our results are consistent with the groove binding model and eliminate the possibility of intercalation in the base pairs of guanine quartet.
\end{abstract}

\section{Introduction}

Telomeres, the end caps on eukaryotic chromosomes, protect chromosomal termini from degradation and fusion. ${ }^{1-3}$ The telomeric DNA sequences generally consist of many tandem repeats of guanine-rich (G-rich) motifs, and various structures can be formed in a natural G-rich DNA sequences. ${ }^{4-5}$ Of particular interest is the quadruplex structures formed by the Hoogsteen hydrogen bond among four guanine bases. ${ }^{6} \mathrm{Re}$ cently, Shafer et al. ${ }^{7}$ have introduced the 3,3'-diethyloxadicarbocyanine iodide (DODCI) molecule to recognize the structure of guanine dimeric hairpin quadruplexes. The DODCI molecule is dissociated into $\mathrm{I}^{-}$anion and $\mathrm{DODC}^{+}$cation in protic solvents. The visible absorption spectrum of $\mathrm{DODC}^{+}$has a maximum at $576 \mathrm{~nm}$ with a weak shoulder at $530-550 \mathrm{~nm}$. Upon addition of dimeric hairpin quadruplex $\left[\mathrm{d}\left(\mathrm{G}_{4} \mathrm{~T}_{4} \mathrm{G}_{4}\right)\right]_{2}$ (abbreviated as HQ), a new peak appears at $534 \mathrm{~nm}$ and a $\sim 5 \mathrm{~nm}$ red shift of the $576 \mathrm{~nm}$ peak results. On the contrary, spectra similar to those of the free $\mathrm{DODC}^{+}$spectrum were found for other structures with G-rich oligonucleotides, including the linear duplex $[\mathrm{d}(\mathrm{CGCGAATTCGCG})]_{2}$ (LD) and the hairpin duplex $\mathrm{d}\left(\mathrm{CGCGT}_{4} \mathrm{CGCG}\right)(\mathrm{HD})$. The emergence of the $534 \mathrm{~nm}$ peak can be considered as a unique signature of the specific binding of the $\mathrm{DODC}^{+}$to the dimeric hairpin quadruplex structure. Furthermore, they proposed that groove binding is more likely the binding mechanism based on the study of absorption, fluorescence, and CD spectra. However, the precise binding modes of the $\mathrm{DODC}^{+}$to the dimeric hairpin quadruplexes remained uncertain. The purpose of this work is to determine the binding modes of $\mathrm{DODC}^{+}$to the HQ.

Recently, we have studied satellite hole $(\mathrm{SH})$ spectra of the BODIPY derivatives ${ }^{8}$ and the 9 -aminoacridine ${ }^{9-11}$ for establishing the relationship between functional group and structural activity. The displacement of the $\mathrm{SH}$ from resonant hole provides the excited state frequency of the Franck-Condon active mode. More importantly, the intensity of the $\mathrm{SH}$ can be significantly enhanced when tuning the burning wavelength into the vibronic transition. This is because each $\mathrm{SH}$ has its own

* To whom correspondence should be addressed. Telephone, 8862-23668231; fax, 8862-2362-0200; e-mail, tcchang@po.iams.sinica.edu.tw.

$\dagger$ Institute of Atomic and Molecular Sciences.

$\div$ Department of Chemistry. electronic-vibrational transition at the burning wavelength, and therefore, it can be viewed as an individual electronic transition. ${ }^{10-11}$ The high-resolution SH spectrum with a free background is very useful in monitoring the localized interactions of DNA - chromophore complexes. ${ }^{12-14}$ In this work, we introduce for the first time the $\mathrm{SH}$ spectrum, along with normal mode calculation, to identify binding modes of the $\mathrm{DODC}^{+}$to the HQ. Furthermore, a binding structure of HQ:DODC ${ }^{+}$is proposed.

\section{Experimental Section}

The experimental setup for the $\mathrm{SH}$ spectrum has been described elsewhere. ${ }^{15}$ Holes were produced by using a dye laser pumped by a mode-locked and Q-switched Nd:YAG laser (Quantronix 416). ${ }^{16}$ The laser emitted trains of $\sim 10$ pulses separated by $13 \mathrm{~ns}$ at a repetition rate of $500 \mathrm{~Hz}$. The absorption spectra were obtained by dispersing the output of a xenon arc lamp (Oriel 66083) through a home-made double-beam spectrometer with a resolution of $\sim 0.03 \mathrm{~nm}$. Extreme care was taken to ensure that the cross section of the probe beam at the sample was inside the burning area.

DODCI was purchased from Exciton, Inc., and used without further purification. Oligonucleotides were purchased from Applied Biosystems. A stock solution of DODCI was prepared in the mixture of 5:4 glycerol:water $\left(\mathrm{Gl}: \mathrm{H}_{2} \mathrm{O}\right)$ solution. The ratio between dye and oligonucleotide in the $\mathrm{Gl}: \mathrm{H}_{2} \mathrm{O}$ solutions is 1:10 with the dye concentration of $\sim 3 \times 10^{-5} \mathrm{M}$ and the buffer concentration of $0.01 \mathrm{M}$ Tris- $\mathrm{HCl}(\mathrm{pH} 7.5)$ and $0.15 \mathrm{M}$ $\mathrm{NaCl}$. Clear glass was normally formed by introducing the sample directly into a Janis dewar from room temperature to $6 \mathrm{~K}$.

\section{Results}

Figure 1 shows the absorption spectra of the free $\mathrm{DODC}^{+}$ and the $\mathrm{DODC}^{+}$mixed with each of the oligonucleotides of $\mathrm{HD}, \mathrm{LD}$, and $\mathrm{HQ}$ doped in $\mathrm{Gl}: \mathrm{H}_{2} \mathrm{O}$ solution at room temperature. The absorption spectra of the free $\mathrm{DODC}^{+}, \mathrm{HD}: \mathrm{DODC}^{+}$, and $\mathrm{LD}: \mathrm{DODC}^{+}$are very similar to the absorption spectra reported by Shafer et al., ${ }^{7}$ which consist of a prominent band at $\sim 580 \mathrm{~nm}$ with a weak shoulder at $\sim 545 \mathrm{~nm}$. However, the distinct peak at $534 \mathrm{~nm}$ observed in their spectrum of HQ:DODC ${ }^{+}$is replaced 


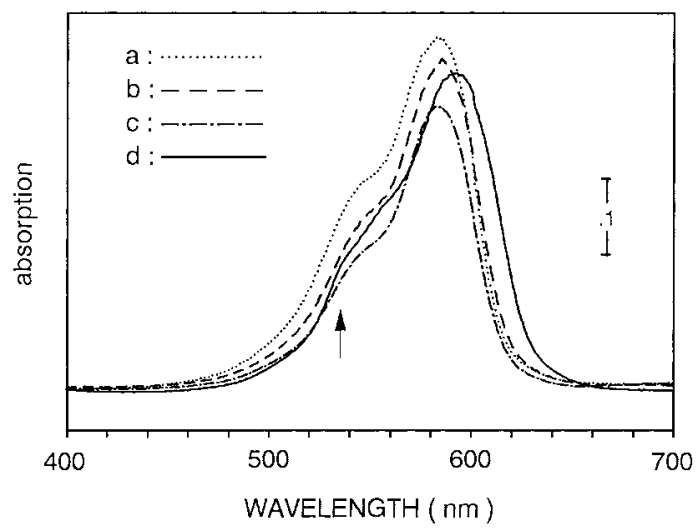

Figure 1. Absorption spectra of $\operatorname{DODC}^{+}$(a), $\mathrm{HD}_{\mathrm{DODC}}^{+}(\mathrm{b}), \mathrm{LD}$ : $\mathrm{DODC}^{+}$(c), and HQ:DODC ${ }^{+}$(d) dissolved in 5:4 glycerl:water solution at room temperature.

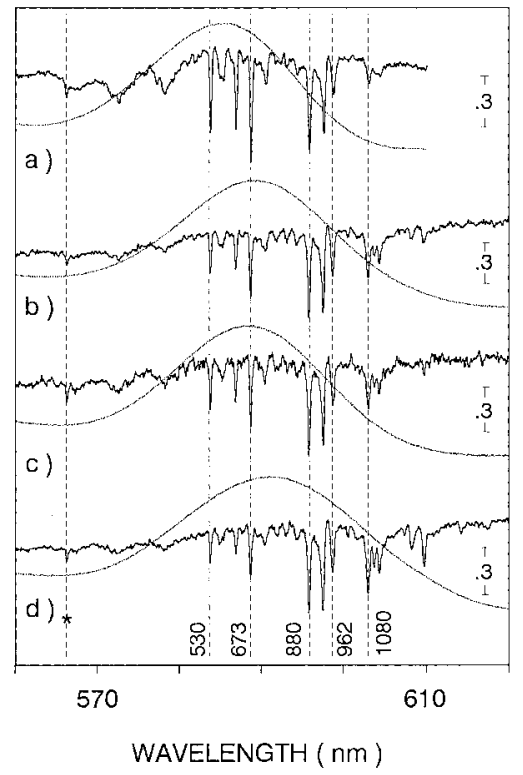

Figure 2. Absorption and SH spectra of $\operatorname{DODC}^{+}$(a), HD:DODC ${ }^{+}$ (b), LD:DODC ${ }^{+}$(c), and HQ:DODC ${ }^{+}$(d) dissolved in 5:4 glycerl:water solution at $\mathrm{T}_{\mathrm{B}} \approx 6 \mathrm{~K}$. The burn wavelength is located at $\lambda_{\mathrm{B}} \approx 566 \mathrm{~nm}$. Prominent satellite holes are labelled with excited-state vibrational frequencies. The intensity of each SH spectrum is multiplied by 10 .

by an additional shoulder at $\sim 535 \mathrm{~nm}$ in our spectrum. The difference between these absorption spectra of $\mathrm{HQ}: \mathrm{DODC}^{+}$may be due to different solution.

Figure 2, plots $\mathrm{a}-\mathrm{d}$, shows the preburn absorption spectra and the $\mathrm{SH}$ spectra of the free $\mathrm{DODC}^{+}, \mathrm{HD}_{\mathrm{DODC}}^{+}, \mathrm{LD}$ : DODC $^{+}$, and HQ:DODC ${ }^{+}$doped in Gl: $\mathrm{H}_{2} \mathrm{O}$ glasses at $T_{\mathrm{B}} \approx 6$ $\mathrm{K}$, respectively. Note that the $\mathrm{SH}$ spectrum is obtained from the difference between each preburn and postburn absorption spectra. The burning wavelength $\left(\lambda_{\mathrm{B}}\right)$ is $\sim 565 \mathrm{~nm}$ with the pulse energy of $\sim 2.5 \mu \mathrm{J}$ and with a burn time of $5 \mathrm{~min}$ for each SH spectrum. Figure 2 shows that the bandwidth of the absorption band of $\mathrm{HQ}: \mathrm{DODC}^{+}$is broader than that of HD: $\mathrm{DODC}^{+}$and LD:DODC ${ }^{+}$by $\sim 30 \%$. However, the normalized hole depths are slightly weaker in the spectra of HQ:DODC ${ }^{+}$ than in those of HD:DODC ${ }^{+}$and LD:DODC ${ }^{+}$.

Figure 3 shows the normalized $\mathrm{SH}$ spectra, the $\mathrm{SH}$ spectrum normalized by preburn absorption spectrum, taken at $\lambda_{\mathrm{B}} \sim 575$ $\mathrm{nm}$ with the same pulse energy of $\sim 2 \mu \mathrm{J}$ and with a burn time of $5 \mathrm{~min}$ for each spectrum. No appreciable difference is found for the $\mathrm{SH}$ frequencies among the spectra of free $\mathrm{DODC}^{+}, \mathrm{LD}$ : $\mathrm{DODC}^{+}$, and HD:DODC ${ }^{+}$. However, the $\mathrm{SH}$ at $163 \mathrm{~cm}^{-1}$ in

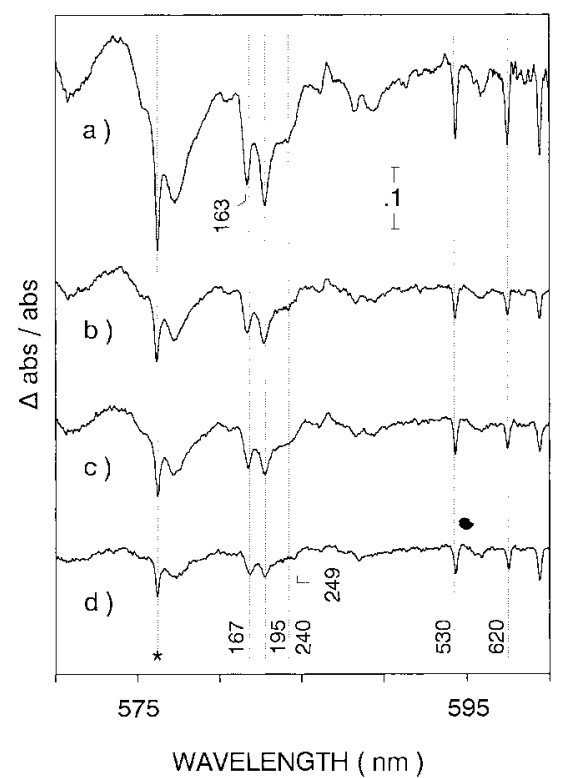

Figure 3. Normalized SH spectra of $\mathrm{DODC}^{+}(\mathrm{a}), \mathrm{HD}: \mathrm{DODC}^{+}(\mathrm{b})$, LD:DODC ${ }^{+}$(c), and HQ:DODC ${ }^{+}$(d) doped in Gl: $\mathrm{H}_{2} \mathrm{O}$ glasses at $\mathrm{T}_{\mathrm{B}}$ $\approx 6 \mathrm{~K}$. The burn wavelength is located at $\lambda_{\mathrm{B}} \approx 576 \mathrm{~nm}$. Prominent satellite holes are labelled with excited-state vibrational frequencies.

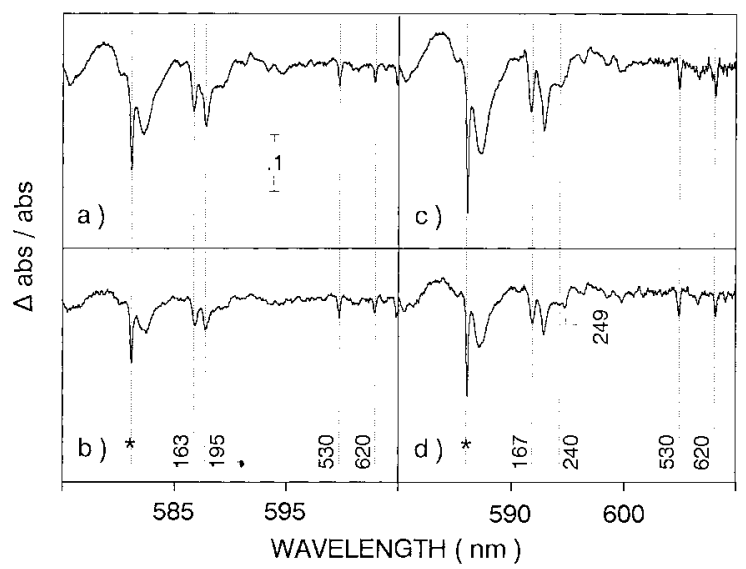

Figure 4. Normalized $\mathrm{SH}$ spectra of HD:DODC ${ }^{+}$(a), HQ:DODC ${ }^{+}$ (b), taken at $\lambda_{\mathrm{B}} \approx 581 \mathrm{~nm}$ and HD:DODC ${ }^{+}$(c), and HQ:DODC ${ }^{+}$(d), taken at $\lambda_{\mathrm{B}} \approx 586 \mathrm{~nm}$ doped in $\mathrm{Gl}_{1} \mathrm{H}_{2} \mathrm{O}$ glasses at $\mathrm{T}_{\mathrm{B}} \approx 6 \mathrm{~K}$. Prominent satellite holes are labelled with excited-state vibrational frequencies.

the spectrum of free DODC ${ }^{+}$is blue-shifted to $167 \mathrm{~cm}^{-1}$ in the spectrum of $\mathrm{HQ}: \mathrm{DODC}^{+}$. In addition, a weak SH at $\sim 240 \mathrm{~cm}^{-1}$ is observed in Figure 3, plots $\mathrm{a}-\mathrm{c}$, but cannot be identified in Figure 3, plot d. On the other hand, a weak SH at $249 \mathrm{~cm}^{-1}$ is definitely observed in the spectrum of HQ:DODC ${ }^{+}$but cannot be identified in other spectra. Another feature is that the depths of the low-frequency SHs are weaker in the spectra of HQ: $\mathrm{DODC}^{+}$than in those of $\mathrm{HD}: \mathrm{DODC}^{+}$and $\mathrm{LD}: \mathrm{DODC}^{+}$. However, the depths of the 530,620, and $673 \mathrm{~cm}^{-1} \mathrm{SHs}$ are similar among them. The weaker hole depths of the lowfrequency SHs may be caused by the red shift of the absorption origin band of $\mathrm{HQ}: \mathrm{DODC}^{+}$, while the burning wavelength is kept constant. Considering the $\sim 5 \mathrm{~nm}$ red shift of the absorption band for HQ:DODC ${ }^{+}$, Figure 4, plots a-d, show the normalized SH spectra of HD:DODC ${ }^{+}$and HQ:DODC ${ }^{+}$taken at $\lambda_{\mathrm{B}} \sim 580$ and $585 \mathrm{~nm}$ with the similar pulse energy of $\sim 2 \mu \mathrm{J}$ and with a burn time of $5 \mathrm{~min}$ for each spectrum, respectively. Figures 3 and 4 show that the shift of the absorption band indeed affects the hole depth. However, the hole depths of the low frequency SHs of HQ:DODC ${ }^{+}$are still weaker than that of HD:DODC ${ }^{+}$. 


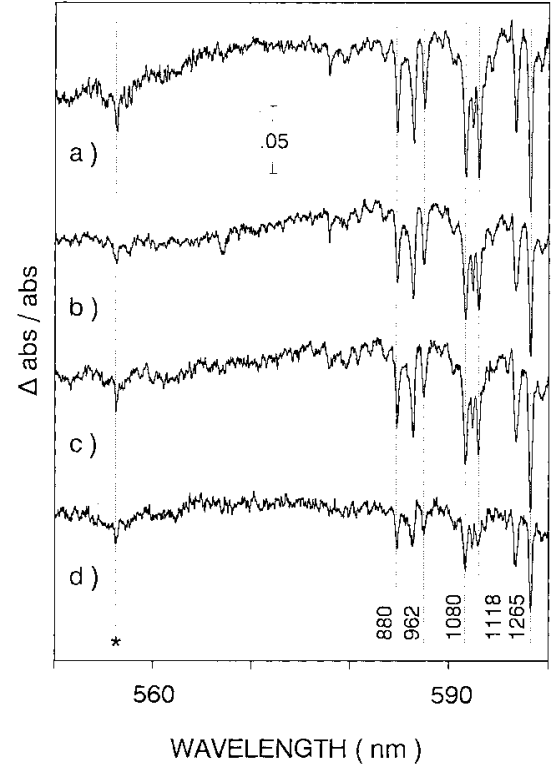

Figure 5. Normalized SH sectra of DODC (a), HD:DODC ${ }^{+}(b)$, LD: $\operatorname{DODC}^{+}(\mathrm{c})$, and HQ:DODC ${ }^{+}(\mathrm{d})$ doped in $\mathrm{Gl}: \mathrm{H}_{2} \mathrm{O}$ glasses at $\mathrm{T}_{\mathrm{B}} \approx 6$ $\mathrm{K}$. The burn wavelength is located at $\lambda_{\mathrm{B}} \approx 556 \mathrm{~nm}$. Prominent satellite holes are labelled with excited-state vibrational frequencies.

Figure 5 shows another set of normalized SH spectra taken at $\lambda_{\mathrm{B}} \sim 555 \mathrm{~nm}$. The same pulse energy of $\sim 2 \mu \mathrm{J}$ is used for each spectrum with the burn time of 5 min for each spectrum. Figure 5 shows no significant difference in these $\mathrm{SH}$ spectra, except the decrease of the hole depth upon interaction with HQ. In particular, the depth of the $1118 \mathrm{~cm}^{-1} \mathrm{SH}$ is further reduced in the spectrum of HQ:DODC ${ }^{+}$.

\section{Mode Assignments}

The assignments of the SHs are essential in identifying the binding modes involved in the HQ:DODC ${ }^{+}$interactions. In

170

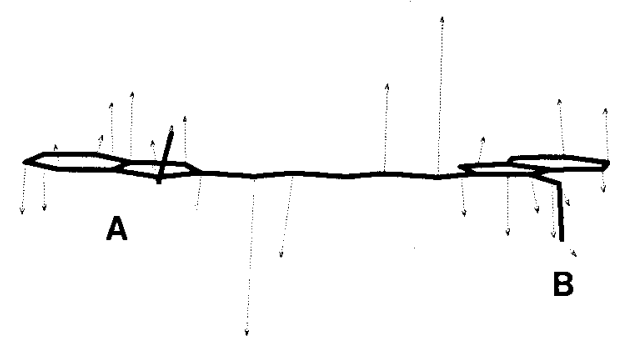

564

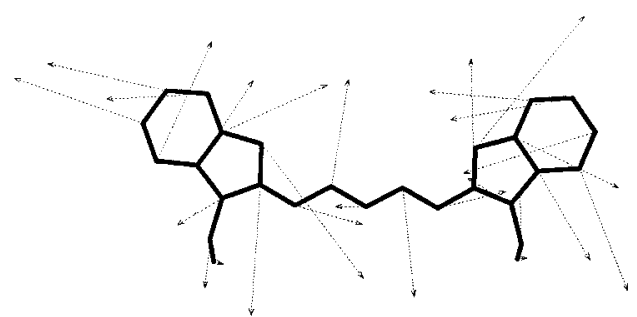

order to distinguish the ring-chromophore, ethyl group, and methine chain modes of DODCI, we have further studied the SH spectra of 3,3'-diethyloxacarbocyanine iodide (DOCI), 3,3'diethylthiadicarbocyanine iodide (DTDCI), and 3,3'-dipropyloxacarbocyanine iodide (DOCI-3) doped in $\mathrm{Gl}: \mathrm{H}_{2} \mathrm{O}$ glass. The mode frequencies higher than $500 \mathrm{~cm}^{-1}$ are compared to the Raman bands of DODC $^{+}$assigned by Gustafson and coworkers. ${ }^{17}$ In addition, Raman spectra of other cyanine dyes are also consulted. ${ }^{18}$ At present, we are not able to find the low-frequency vibrational study of $\mathrm{DODC}^{+}$. AM1 semiempirical calculations in vacuo by a Hyperchem package were conducted for the qualitative assignment of vibrational modes. The conformational geometry of $\mathrm{DODC}^{+}$is first optimized by the Polak-Ribiere algorithm and then applied to perform normal mode calculation. Restricted Hartree-Fock wave functions are used. The calculated diagrams of the $170,564,887$, and 1164 $\mathrm{cm}^{-1}$ modes of DODC ${ }^{+}$are shown in Figure 6.

Our SH spectra show that the 136 and $228 \mathrm{~cm}^{-1} \mathrm{SHs}$ of $\mathrm{DOC}^{+}$differ from the 163 and $240 \mathrm{~cm}^{-1} \mathrm{SHs}_{\text {of }} \mathrm{DODC}^{+}$. Note that the $167 \mathrm{~cm}^{-1}$ mode of 1,3,5-hexatriene was assigned to $\mathrm{C}-\mathrm{C}-\mathrm{C}$ deformation of the polymethine chain. ${ }^{19}$ In addition, it is known that some out-of-plane vibrations of the retinal chain appear below $500 \mathrm{~cm}^{-1} .^{20}$ AM1 calculations suggest that the 170 and $243 \mathrm{~cm}^{-1}$ modes are mainly attributed to methine chain motion. The $530 \mathrm{~cm}^{-1} \mathrm{SH}$ of $\mathrm{DODC}^{+}$is consistent with the $527 \mathrm{~cm}^{-1} \mathrm{SH}$ of DTDC ${ }^{+}$, but differs from the $558 \mathrm{~cm}^{-1} \mathrm{SH}$ of $\mathrm{DOC}^{+}$. Sato et al. ${ }^{18}$ have tentatively assigned the band near $550 \mathrm{~cm}^{-1}$ of some carbocyanine dyes to the bending vibration of methine chain. AM1 calculation shows that the $564 \mathrm{~cm}^{-1}$ mode involves the coupling of ring and chain motions. The 885 and $1078 \mathrm{~cm}^{-1} \mathrm{SHs}$ of $\mathrm{DOC}^{+}$are very similar to the 880 and $1080 \mathrm{~cm}^{-1} \mathrm{SHs}$ of DODC ${ }^{+}$. AM1 calculations show that the 887 and $1085 \mathrm{~cm}^{-1}$ modes are due to ring vibrations. In addition, Gustafson et al. ${ }^{17}$ have assigned the 884 and $1088 \mathrm{~cm}^{-1}$ Raman bands of $\mathrm{DODC}^{+}$to ring vibrations. Furthermore, the approximately identical frequency at $1118 \mathrm{~cm}^{-1} \mathrm{SH}$ observed in the spectra of $\mathrm{DOC}^{+}$and $\mathrm{DODC}^{+}$infers that this mode is

887

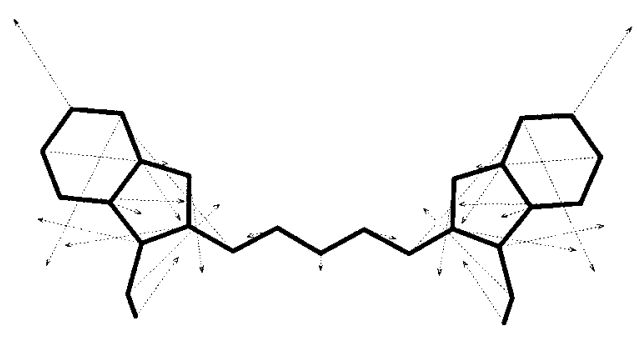

1164

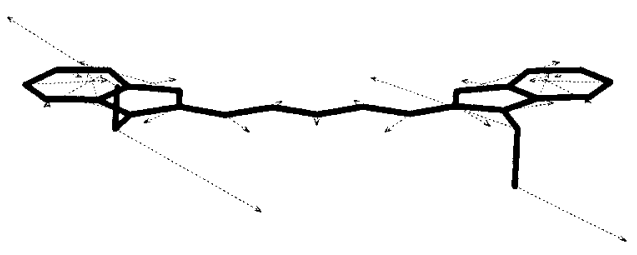

Figure 6. Schematic diagrams of the $170,564,887$, and $1164 \mathrm{~cm}^{-1}$ modes of DODC ${ }^{+}$. 
TABLE 1: Mode Assignments of Prominent Satellite Holes of DODC ${ }^{+}$

\begin{tabular}{ll}
\hline \multicolumn{1}{c}{ DODC $^{+}$} & \multicolumn{1}{c}{ assignment } \\
\hline 163,240 & methine chain \\
530 & chain and ring coupling \\
$880,962,1080$ & ring chromophore \\
1118 & ethyl group
\end{tabular}

most likely due to end chromophore motion. However, Sato et al. ${ }^{18}$ have assigned the $1135 \mathrm{~cm}^{-1}$ band of $\mathrm{DTDC}^{+}$to the vibration associated with the ethyl group. In order to test this assignment, we have studied the SH spectrum of DOCI-3. The disappearance of the $1118 \mathrm{~cm}^{-1} \mathrm{SH}$ and the occurrence of a very weak $\mathrm{SH}$ at $1130 \mathrm{~cm}^{-1}$ in the spectrum of DOCI-3 support the assignment of $1118 \mathrm{~cm}^{-1} \mathrm{SH}$ of $\mathrm{DODC}^{+}$to the ethyl vibration. The mode assignments of these SHs of $\mathrm{DODC}^{+}$are listed in Table 1.

\section{Discussion}

The additional shoulder at $535 \mathrm{~nm}$, the significant red shift, and the largest width of the absorption band of HQ:DODC ${ }^{+}$ among these DNA:DODC ${ }^{+}$complexes indicate that the $\mathrm{DODC}^{+}$ is more perturbed by the HQ interactions. Shafer et al. ${ }^{7}$ have suggested that structural differences among HD, LD, and HQ are responsible for the specific dye recognition. This is because the HD and LD have two different grooves, but HQ has three different grooves characterized by wide, medium, and narrow grooves. ${ }^{21}$ The broader bandwidth of the absorption band of $\mathrm{HQ}: \mathrm{DODC}^{+}$can be described by more environmental interactions resulting from the additional groove of HQ structure. In addition, various widths among these grooves are determined by different orientation of backbone phosphate groups. ${ }^{22}$ The red shift of the absorption band of $\mathrm{HQ}: \mathrm{DODC}^{+}$suggests that a specific groove of HQ can possibly better interact with $\mathrm{DODC}^{+}$ since the red shift of the $\pi-\pi^{*}$ transition band is usually attributed to an increase in environmental polarity. ${ }^{23}$

Besides the different absorption spectral features of HQ: $\mathrm{DODC}^{+}$, the vibrational spectra can provide the information of the coupling modes of DODC ${ }^{+}$to the HQ. Very similar spectral features, including frequencies and relative hole depths, of $\mathrm{SHs}$ among the free $\mathrm{DODC}^{+}, \mathrm{HD}: \mathrm{DODC}^{+}$, and LD:DODC ${ }^{+}$suggest that there is no specific binding of $\mathrm{DODC}^{+}$to the $\mathrm{HD}$ and LD. However, the frequency shift of the SH from $163 \mathrm{~cm}^{-1}$ to 167 $\mathrm{cm}^{-1}$ and the different features at $\sim 240 \mathrm{~cm}^{-1} \mathrm{SH}$ upon interaction with $\mathrm{HQ}$ infer that specific coupling is involved for the chain moiety of $\mathrm{DODC}^{+}$to the HQ. Considering the electrostatic interaction between the phosphate group of the specific groove and the positive charge delocalized on the conjugated chain moiety of $\mathrm{DODC}^{+}$, the chain motions of $\mathrm{DODC}^{+}$can be more perturbed by the HQ interactions. It is very possible that the chain moiety of $\mathrm{DODC}^{+}$is trapped in the specific groove of HQ. In addition, the depths of the lowfrequency SHs of HQ:DODC ${ }^{+}$are weaker than those of HD: $\mathrm{DODC}^{+}$, but the depths of the 530 and $620 \mathrm{~cm}^{-1}$ SHs are very similar between them, supporting the claim that the chain moiety of $\mathrm{DODC}^{+}$is a coupling site to the HQ.

Furthermore, the approximately identical hole depths at 530, 620 , and $673 \mathrm{~cm}^{-1}$ in the SH spectra of $\mathrm{HD}: \mathrm{DODC}^{+}, \mathrm{LD}$ : $\mathrm{DODC}^{+}$, and $\mathrm{HQ}: \mathrm{DODC}^{+}$suggest that $\mathrm{DODC}^{+}$cannot be located in the pocket of the quartet of HQ. This feature also eliminates the possibility of intercalation in the base pairs of guanine quartet. Our previous study of 9-aminoacridine indicated that the hole depth of 9-AA is significantly reduced to less than $1 / 10$ upon intercalation in DNA. ${ }^{14}$ In addition, the study of thionine shows similar reduction of the hole depth upon intercalation with calf thymus. ${ }^{24}$ Our results support that the groove binding plays an important role between $\mathrm{HQ}$ and $\mathrm{DODC}^{+}$interaction.

The hole depths of the ring modes of HQ are weaker than that of $\mathrm{HD}$ and $\mathrm{LD}$, implying that the interaction of the ring chromophore of DODC ${ }^{+}$to the HQ differs from that to the HD and LD. The decrease of the hole depth on the ring modes of $\mathrm{DODC}^{+}$is consistent with the proposed binding of the $\mathrm{DODC}^{+}$ to the $\mathrm{HQ}$, with one end chromophore A (for simplicity, we mark the two end chromophores as A and B) projecting into the thymine loop region and stacking between the loop thymines and the terminal guanine quartet. ${ }^{7}$ Another interesting feature is the increased reduction in depth of the $1118 \mathrm{~cm}^{-1} \mathrm{SH}$ peak in Figure 5d, implying that the coupling of the ethyl group of $\mathrm{DODC}^{+}$to the HQ can be strong. It further suggests that the end ring $\mathrm{B}$ has less interaction with $\mathrm{HQ}$. More experiments are necessary to examine how the ethyl group of $\mathrm{DODC}^{+}$ interacts with HQ and how the chain length of $\mathrm{DODC}^{+}$affects the recognition behavior.

In summary, we have illustrated that by using the highresolution satellite holes, especially including the low-frequency satellite holes, along with the characteristics of particular vibrations of $\mathrm{DODC}^{+}$, we are able to identify the local interactions of various structures of G-rich DNA. Our SH results are consistent with groove binding model and eliminate the possibility of ring intercalation in the base pairs of guanine quartet. The $\mathrm{SH}$ spectroscopic finding points to an interesting direction, namely the microscopic study of the local interactions of chromophores to oligonucleotides. The study of the binding modes and binding structures for the recognition of G-quartet should be important for our better understanding of the telomeres.

Acknowledgment. This work was supported by the Academia Sinica and the NSC of the Republic of China (Grant NSC86-2113-M001-045). Chang is grateful for the useful comments and suggestions from referees.

\section{References and Notes}

(1) Blackburn, E. H., Greider, C. W., Eds. Telomeres; Cold Spring Harbor Laboratory Press: Cold Spring Harbor, New York, 1996.

(2) Zakian, V. A. Science 1995, 270, 1601 703.

(3) Williamson, J. R. Annu. Rev. Biophys. Biomol. Struct. 1994, 23,

(4) Gellert, M.; Lipsett, M. N.; Davies, D. R. Proc. Natl. Acad. Sci. U.S.A. 1962, 48, 2013.

(5) Sen, D.; Gilbert, W. Nature 1988, 334, 364

(6) Fang, G.; Cech, T. R. Cell 1993, 74, 875.

(7) Chen, Q.; Kuntz, I. W.; Shafer, R. H. Proc. Natl. Acad. Sci. U.S.A. 1996, 93, 2635.

(8) Chiang, C. C.; Wang, J. H.; Cheng, J. Y.; Chang, T.-C. Chem Phys. Lett. 1995, 239, 95.

(9) Chiang, C. C.; Cheng, J. Y.; Cheng, Y. R.; Chen, H. S.; Mou, C Y.; Chang, T.-C. Mol. Cryst. Liq. Cryst. 1996, 291, 175.

(10) Chiang, C. C.; Hwang, B. C.; Yu, J.; Cheng, J. Y.; Mou, C. Y.; Lin, S. H.; Chang, T.-C. J. Chem. Soc., Faraday Trans. 1997, 93, 1297.

(11) Chiang, C. C.; Mou, C. Y.; Chang, T.-C. Chem. Phys. Lett. 1997, 273,153 .

(12) Chang, T. C.; Chiang, C. C.; Peck, K. J. Am. Chem. Soc. 1995, 117, 7576.

(13) Chang, T.-C.; Chiang, C. C.; Peck, K. In Trends in Optics and Photonics: Biomedical Optical Spectroscopy and Diagnostics; SevickMuraca, E., Benaron, D., Eds.; Optical Society of America: Washington, DC, 1996

(14) Cheng, J. Y.; Chiang, C. C.; Cheng, Y. R.; Chen, H. S.; Lin, L. J.; Chang, T.-C. J. Chin. Chem. Soc. 1997, 44, 97.

(15) Chang, T.-C.; Chiang, C. C.; Small, G. J.; Chou, S. H. Chem. Phys. Lett. 1994, 223, 190.

(16) Chang, T.-C.; Jou, B. H.; Ou, R. H.; Chiang, C. C.; Li, H. W. Chem. Phys. Lett. 1991, 187, 208. 
(17) Iwata, K.; Weaver, W. L.; Gustafson, T. L. J. Phys. Chem. 1992, 96, 10219.

(18) Sato, H.; Kawasaki, M.; Kasatani, K.; Katsumata, M. J. Raman Spectrosc. 1988, 19, 129.

(19) Keszthelyi, T.; Wilbrandt, R.; Cave, R. J.; Johnson, J. L. J. Phys. Chem. 1994, 98, 5632 .

(20) Curry, B.; Broek, A.; Lugtenburg, J.; Mathies, R. J. Am. Chem. Soc. 1982, 104, 5274.
(21) Smith, F. W.; Feigon, J. Nature 1992, 356, 164.

(22) Laughlan, G.; Murchie, A. I. H.; Norman, D. G.; Moore, M. H.; Moody, P. C. E.; Lilley, D. M. J.; Luisi, M. Science 1994, 265, 520.

(23) Turro, N. J. Modern Molecular Photochemistry; Benjamin/Cummings Publishing Co.: Menlo Park, CA, 1978.

(24) Weng, C. I.; Chiang, C. C.; Cheng, J. Y.; Chang, T.-C. In preparation. 